\title{
MOVING MESH FOR THE AXISYMMETRIC HARMONIC MAP FLOW
}

\author{
Benoit Merlet ${ }^{1}$ And Morgan Pierre ${ }^{2,3}$
}

\begin{abstract}
We build corotational symmetric solutions to the harmonic map flow from the unit disc into the unit sphere which have constant degree. First, we prove the existence of such solutions, using a time semi-discrete scheme based on the idea that the harmonic map flow is the $\mathrm{L}^{2}$-gradient of the relaxed Dirichlet energy. We prove a partial uniqueness result concerning these solutions. Then, we compute numerically these solutions by a moving-mesh method which allows us to deal with the singularity at the origin. We show numerical evidence of the convergence of the method.
\end{abstract}

Mathematics Subject Classification. 35A05, 35K55, 65N30, 65N50, 65N99.

Received: June 10, 2004. Revised: March 2, 2005.

\section{INTRODUCTION}

Let $D$ be the unit disc in $\mathbb{R}^{2}$ and let $\mathbf{S}^{2}$ be the two dimensional unit sphere in $\mathbb{R}^{3}$. In this paper, we compute solutions $u: \bar{D} \times[0,+\infty) \rightarrow \mathbf{S}^{2}$ to the harmonic map flow:

$$
\begin{array}{rll}
u_{t}=\Delta u+u|\nabla u|^{2} & \text { in } & D \times(0,+\infty), \\
u=u_{0} & \text { on } & \partial D \times[0,+\infty), \\
u=u_{0} & \text { on } & D \times\{0\},
\end{array}
$$

where the initial condition $u_{0}$ is a map from $\bar{D}$ to $\mathbf{S}^{2}$. The harmonic map heat flow has been introduced by Eells and Sampson in [7] as the $\mathrm{L}^{2}$ gradient of the Dirichlet energy:

$$
\mathcal{E}(u)=\frac{1}{2} \int_{D}|\nabla u|^{2} .
$$

A result of Struwe [15], completed by Chang [6] for domains with boundary, asserts that if $u_{0}$ belongs to $\mathrm{H}^{1}\left(D, \mathbf{S}^{2}\right)$ with $u_{0 \mid \partial D}$ in $\mathrm{H}^{3 / 2}\left(\partial D, \mathbf{S}^{2}\right)$, then problem (1)-(3) has a weak solution $u \in \mathrm{H}_{l o c}^{1}(\bar{D} \times[0,+\infty))$, which is smooth away from finitely many singular points $\left(x_{i}, t_{i}\right)_{1 \leq i \leq N} \subset \bar{D} \times[0,+\infty)$. Its Dirichlet energy

\footnotetext{
Keywords and phrases. Moving mesh, finite elements, harmonic map flow, axisymmetric.

1 ENS Cachan, Antenne de Bretagne, Avenue Robert Schumann, 35170 Bruz France.

benoit.merlet@bretagne.ens-cachan.fr

2 Laboratoire de Mathématiques, Université de Poitiers, Bd Marie et Pierre Curie, BP 30179, 86962 Futuroscope Cedex, France. Morgan.Pierre@math.univ-poitiers.fr

3 Centre de Mathématiques et de Leurs Applications, École Normale Supérieure de Cachan, 61 Av. du Président Wilson, 94235 Cachan Cedex, France.

(c) EDP Sciences, SMAI 2005
} 
$\mathcal{E}(u(t))$ is non-increasing and Freire [9] showed that this monotonicity guarantees the uniqueness in the class $\mathrm{H}_{l o c}^{1}\left(\bar{D} \times[0,+\infty), \mathbf{S}^{2}\right)$. This solution is usually referred to as the "Struwe solution".

The behaviour of the Struwe solution near a singularity has been described by Qing [13]. Briefly, at such a point, the energy $\mathcal{E}(u(t))$ has a jump which corresponds to a concentration phenomenon called bubbling of spheres. The reverse phenomenon (debubbling) has been used in $[3,16]$ to build a weak solution to (1)-(3) in $\mathrm{H}_{l o c}^{1}(\bar{D} \times[0,+\infty))$ different from the Struwe solution. The idea is to keep in mind the energy lost at a bubbling time $t_{1}$ and to release it at some time $t_{2}>t_{1}$ for a debubbling (the energy has an upward jump at time $t_{2}$ ). When a bubbling or a debubbling occurs, the $\mathbf{S}^{2}$-degree of the map $u(t)$, defined by

$$
\operatorname{deg}_{\mathbf{S}^{2}}(u):=\frac{1}{4 \pi} \int_{D}\left(u_{x} \times u_{y}\right) \cdot u
$$

may also jump, because the limit of a $\mathrm{H}^{1}$-weakly converging sequence does not in general have the same degree as the elements of the sequence [4].

In the following, we only consider corotational symmetric maps, i.e. maps $u: D \rightarrow \mathbf{S}^{2}$ such that

$$
u(r \cos \varphi, r \sin \varphi)=(\cos \varphi \cos \theta(r), \sin \varphi \cos \theta(r), \sin \theta(r))
$$

for some $\theta:[0,1] \times[0,+\infty) \rightarrow \mathbb{R}$. The examples constructed by Bertsch et al. [3] and Topping [16] to prove the non-uniqueness of weak solution to (1)-(3) were actually corotational symmetric maps. In terms of $\theta$, equations (1)-(3) are equivalent to

$$
\begin{aligned}
\theta_{t}=\theta_{r r}+\frac{1}{r} \theta_{r}+\frac{\sin (2 \theta)}{2 r^{2}} \quad & \text { in } \quad(0,1) \times(0,+\infty), \\
\theta(1, t)=\theta_{0}(1) & \text { for } \quad t \geq 0 \\
\theta(r, 0)=\theta_{0}(r) \quad \text { for } \quad & 0 \leq r \leq 1
\end{aligned}
$$

where $\theta_{0}$ is associated to a corotational initial data $u_{0}$. The Dirichlet energy is given by

$$
\mathcal{E}(\theta):=\mathcal{E}(u)=\pi \int_{0}^{1}\left(\frac{\cos ^{2} \theta}{r}+\theta_{r}^{2} r\right) \mathrm{d} r .
$$

Now introduce the set of functions with bounded energy

$$
\mathcal{S}_{\alpha}:=\left\{\theta \in \mathrm{C}^{0}([0,1], \mathbb{R}), \theta(1)=\alpha, \frac{\cos \theta}{\sqrt{r}} \in \mathrm{L}^{2}(] 0,1[) \text { and } \sqrt{r} \theta^{\prime} \in \mathrm{L}^{2}(] 0,1[)\right\}
$$

where $\alpha=\theta_{0}(1) \in \mathbb{R}$ corresponds to a boundary condition on $\partial D$. Every $\theta \in \mathcal{S}_{\alpha}$ satisfies

$$
\theta(0)=-\frac{\pi}{2}+k \pi
$$

where $k \in \mathbb{Z}$ is (up to a factor 2) the winding number of $\theta$, and characterizes the corotational degree of $\theta$ defined in Section 1. In this paper, we build solutions to (5)-(7) with constant corotational degree. We believe these solutions to be the ones described by Bertsch et al. in the introduction of [3]. This approach seems to be more accurate for the description of nematic liquid crystals. Unfortunately, at this time, we are not able to give a rigorous characterization of our solutions and then to ensure uniqueness. However, we shall explain physically the desired properties for these solutions and compare them to the Struwe solutions. 


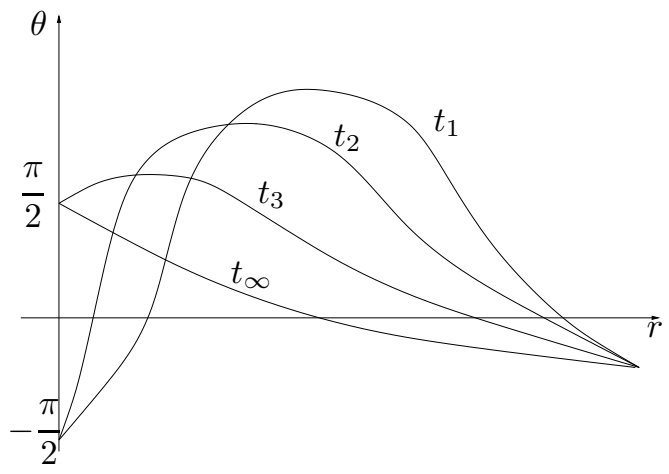

Figure 1. Struwe solution.

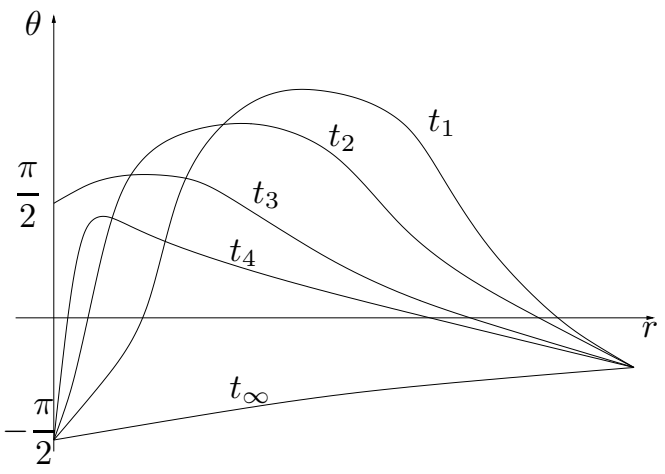

Figure 2. Reverse bubbling.

Figure 1 illustrates a Struwe solution $\bar{\theta}(r, t)$ at several times $0=t_{1}<t_{2}<t_{3}<t_{\infty}=+\infty$. The initial boundary condition at $r=0$ is $\bar{\theta}(0, t)=-\pi / 2$. This solution bubbles at some time $\bar{t}$ in $\left(t_{2}, t_{3}\right)$ and the boundary condition becomes $\bar{\theta}(0, t)=+\pi / 2$ for $t \geq \bar{t}$. The energy $\mathcal{E}(\bar{\theta}(t))$ is nonincreasing and is smooth away from $t=\bar{t}$, but $\lim _{t \rightarrow \bar{t}^{-}} \mathcal{E}(\bar{\theta}(t))=\mathcal{E}(\bar{\theta}(\bar{t}))+4 \pi$.

For nematic liquid crystals, it is reasonable to expect that the energy and the degree are preserved during the bubbling. A way to formulate this idea is to consider that every $\theta \in \mathcal{S}_{\alpha}$ satisfies the prescribed boundary condition $\theta(0)=-\pi / 2$ at $r=0$ even if $\theta\left(0^{+}\right)=\lim _{r \rightarrow 0} \theta(r)$ is different from this value. In other words, we add to the graph of $\theta$ in $[0,1] \times \mathbb{R}$ the vertical segment joining $(0,-\pi / 2)$ to $\left(0, \theta\left(0^{+}\right)\right)$, which contains the energy $4\left|\theta\left(0^{+}\right)+\pi / 2\right|$. The total energy,

$$
\mathcal{E}_{0}(\theta):=\mathcal{E}(\theta)+4\left|\theta\left(0^{+}\right)+\pi / 2\right|
$$

will be defined more generally in Section 1 . We seek solutions with nonincreasing energy $\mathcal{E}_{0}(\theta(t))$ rather than nonincreasing $\mathcal{E}(\theta(t))$.

Figure 2 represents such a solution $\underline{\theta}(r, t)$, with the same initial data as $\bar{\theta}$. There exists a time $\underline{t}$ in $\left(t_{3}, t_{4}\right)$ such that $\underline{\theta}(r, t)=\bar{\theta}(r, t)$ for $0 \leq t \leq \underline{t}, \underline{\theta}_{r}(0, \underline{t})=0$, and $\underline{\theta}(r, \underline{t})<\pi / 2$ for $r>0$ small. It is possible to decrease the energy $\mathcal{E}_{0}(\underline{\theta}(t))$ by a debubbling. The energy of the vertical part is released and $\underline{\theta}(0, t)=-\pi / 2$ for $t>\underline{t}$. The energy $\mathcal{E}_{0}(\underline{\theta}(t))$ is continuous and nonincreasing. In this example, no other bubbling or debubbling occurs, and $\lim _{t \rightarrow+\infty} \underline{\theta}(\cdot, t) \neq \lim _{t \rightarrow+\infty} \bar{\theta}(\cdot, t)$. Notice that we could have chosen a debubbling time $t>\underline{t}$, but we look for solutions debubbling at the first possible time.

Our construction of solutions to (5)-(7) with prescribed degree $\theta(0)=-\pi / 2+k \pi$ is a time semi-discrete scheme introduced by Bethuel et al. [2] for domains in $\mathbb{R}^{3}$. It is a backward Euler scheme based on the idea that the harmonic map flow is the $\mathrm{L}^{2}$-gradient of $\mathcal{E}_{0}$ (replacing $\mathcal{E}_{0}$ with $\mathcal{E}$, we think that we would obtain the Struwe solution). In addition, the total energy $\mathcal{E}_{0}\left(\theta^{\tau}(t)\right)$ of the solution $\theta^{\tau}$ to the semi-discrete scheme is nonincreasing.

For the numerical simulation of (5)-(7), in order to deal with the singularity at $r=0$, we use a moving-mesh method based on a piecewise linear $\left(P_{1}\right)$ finite element discretization, as introduced in [1] for the stationary case. Moving-mesh methods consist in moving the nodes of a given initial mesh to new locations, the number and connectivity of nodes being fixed (see [5] and references therein). For steady-state problems in which the true solution minimizes a functional, the idea is to look for the nodes that minimize the functional obtained by a finite element discretization $[10,14]$ ("moving finite elements"). In our case, the minimization problem is obtained by the time semi-discrete scheme associated to (5)-(7). As in the steady-state case, the discrete minimizer exists for a relaxed discrete functional, and its graph may have a vertical part at $r=0$. 
The paper is organized as follows. In Section 1, we recall the main results concerning the steady-state case. In Section 2, we obtain the existence of weak solutions to (5)-(7) with prescribed degree by proving the convergence of the time semi-discrete scheme; we also prove a partial uniqueness result. Section 3 concerns the space discretization of the time semi-discrete scheme by $P_{1}$ moving finite elements; we establish a convergence result. We discuss computational aspects in Section 4; in particular, we explain the difference between the implemented algorithm, based on a Gaussian quadrature, and the ideal algorithm from Section 3. Before presenting in Section 6 the numerical results, we show numerical evidence of the convergence in Section 5 .

\section{Mathematical Setting}

In this section, we sum up the results in [1] concerning the steady-state problem for corotational symmetric maps. The concentration phenomenon is necessarily located at $r=0$. For a regular corotational map $u$, the $\mathbf{S}^{2}$-degree (4) is equal to $\operatorname{deg}_{\mathbf{S}^{2}}(u)=-(1 / 2) \int_{\theta(0)}^{\theta(1)} \cos \varphi \mathrm{d} \varphi$. We define the corotational $\mathbf{S}^{2}$-degree as

$$
\operatorname{cor} \operatorname{deg}_{\mathbf{S}^{2}}(\theta):=-\frac{1}{2} \int_{\theta(0)}^{\theta(1)}|\cos \varphi| \mathrm{d} \varphi
$$

where $\theta$ is associated to the map $u$. The latter is closely related to the $S^{1}$-degree $\theta(1)-\theta(0)$ of $u$ : if $\theta(1)-\theta(0)=2 k \pi$, then $\operatorname{cor} \operatorname{deg}_{\mathbf{S}^{2}}(\theta)=-2 k$ where $k$ is the winding number of $u_{\mid[0,1]}$. The fundamental property is that the energy controls the degree:

$$
4 \pi\left|\operatorname{cor} \operatorname{deg}_{\mathbf{S}^{2}}(\theta)\right| \leq \mathcal{E}(\theta),
$$

and equality occurs if and only if $\theta$ corresponds to a corotational harmonic map [1] (that is a critical point of the Dirichlet energy).

Relation (8) gives a partition of $\mathcal{S}_{\alpha}$ into homotopy classes $\mathcal{H}_{k, \alpha}$, where

$$
\mathcal{H}_{k, \alpha}:=\left\{\theta \in \mathrm{C}^{\infty}([0,1], \mathbb{R}), \theta(0)=-\frac{\pi}{2}+k \pi, \theta(1)=\alpha\right\} .
$$

Recall the gap phenomenon [4]: the infimum $\inf \left\{\mathcal{E}(\theta) ; \theta \in \mathcal{H}_{k, \alpha}\right\}$ is not reached in general. We will say that a sequence $\left(\theta_{n}\right)$ converges weakly to $\theta$ in $\mathcal{S}_{\alpha}$ if $\sup _{n} \mathcal{E}\left(\theta_{n}\right)<+\infty$ and $\theta_{n} \rightarrow \theta$ in $\mathcal{D}^{\prime}(0,1)$. For $\theta \in \mathcal{S}_{\alpha}$, the relaxed energy associated to $\mathcal{H}_{k, \alpha}$ is defined as the natural lower semi-continuous extension of $\mathcal{E}_{\mid \mathcal{H}_{k, \alpha}}$ in $\mathcal{S}_{\alpha}$,

$$
\begin{aligned}
\mathcal{E}_{k}(\theta) & :=\inf \left\{\liminf _{i \rightarrow+\infty} \mathcal{E}\left(\theta_{i}\right) ; \theta_{i} \in \mathcal{H}_{k, \alpha}, \theta_{i} \underset{i \rightarrow+\infty}{\rightarrow} \theta \text { in } \mathcal{D}^{\prime}(0,1)\right\} \\
& =\mathcal{E}(\theta)+4\left|\theta\left(0^{+}\right)-k \pi+\frac{\pi}{2}\right| .
\end{aligned}
$$

The interest of the relaxed energy is the following:

Theorem 1.1 (Alouges and Pierre [1]). There exists a unique minimizer for $\mathcal{E}_{k}$ in the class $\mathcal{S}_{\alpha}$, and

$$
\inf _{\theta \in \mathcal{S}_{\alpha}} \mathcal{E}_{k}(\theta)=\inf _{\theta \in \mathcal{H}_{k, \alpha}} \mathcal{E}(\theta)
$$




\section{Time DiscretizATION}

For the time discretization of problem (5)-(7), we use a backward Euler scheme introduced by Bethuel et al. [2] for domains in $\mathbb{R}^{3}$. We adapt it to the corotational case with the condition on the degree $k$.

More precisely, let $\tau>0$ denote the time-step, the algorithm is as follows. We set $\theta^{0}=\theta_{0} \in \mathcal{S}_{\alpha}$ and we assume that $\theta^{n} \in \mathcal{S}_{\alpha}, n \geq 0$ is known. The next step is obtained by:

$$
\theta^{n+1} \text { minimizes } \mathcal{E}_{k}(\theta)+\frac{\pi}{\tau} \int_{0}^{1}\left|\theta-\theta^{n}\right|^{2} r \mathrm{~d} r \text { in } \mathcal{S}_{\alpha} .
$$

From the lower semi-continuity of $\mathcal{E}_{k}[1]$ and the weak compactness of bounded sequences in $\mathcal{S}_{\alpha}$, we know that problem (11) has at least one solution $\theta^{n+1}$, which satisfies the Euler-Lagrange equation

$$
r\left(\frac{\theta^{n+1}-\theta^{n}}{\tau}\right)=\left(r \theta_{r}^{n+1}\right)_{r}+\frac{\sin \left(2 \theta^{n+1}\right)}{2 r} \quad \text { in } \mathcal{D}^{\prime}((0,1)) .
$$

The sequence $\left(\theta^{n}\right)_{n}$ may not be uniquely defined. From the discrete values $\theta_{0}, \theta_{1}, \ldots$, we construct two functions $\theta^{\tau}, \bar{\theta}^{\tau}:[0,1] \times[0, \infty)$ by setting for all $n \in \mathbb{N}$ and $t \in[n,(n+1) \tau)$ :

$$
\begin{aligned}
& \theta^{\tau}(\cdot, t)=\frac{(n+1) \tau-t}{\tau} \theta^{n}+\frac{t-n \tau}{\tau} \theta^{n+1}, \\
& \bar{\theta}^{\tau}(\cdot, t)=\theta^{n} .
\end{aligned}
$$

Notice that $\theta^{\tau}(\cdot, t) \notin \mathcal{S}_{\alpha}$ in general. Indeed, if $\theta^{n}(0) \neq \theta^{n+1}(0)$ then $\theta^{\tau}(0, t) \notin \frac{\pi}{2}+\pi \mathbb{Z}$ for almost every $t \in(n \tau,(n+1) \tau)$. The definition of $\theta^{n+1}$ implies:

$$
\mathcal{E}_{k}\left(\theta^{n+1}\right)+\frac{\pi}{\tau} \int_{0}^{1}\left|\theta^{n+1}-\theta^{n}\right|^{2} r \mathrm{~d} r \leq \mathcal{E}_{k}\left(\theta^{n}\right) .
$$

Summing these inequalities for $n=0 \ldots N-1$, we get the following bound:

$$
\mathcal{E}_{k}\left(\bar{\theta}^{\tau}(\cdot, N \tau)\right)+\pi \int_{0}^{N \tau} \int_{0}^{1}\left|\theta_{t}^{\tau}\right|^{2} r \mathrm{~d} r \mathrm{~d} t \leq \mathcal{E}_{k}\left(\theta_{0}\right)
$$

We now consider every map $\theta$ on $(0,1)$ as an axisymmetric function on $D$ defined by $\theta(r \cos \varphi, r \sin \varphi):=\theta(r)$. With this identification, $\mathrm{L}^{2}(0,1 ; r \mathrm{~d} r)$ is isomorphic to $\mathrm{L}_{a x i}^{2}(D)$, which we define as the closure in $\mathrm{L}^{2}(D)$ of regular axisymmetric functions. Similarly, the Hilbert space $\left\{\theta \in \mathrm{L}^{2}(r \mathrm{~d} r) ; \theta_{r} \in \mathrm{L}^{2}(r \mathrm{~d} r)\right\}$, equipped with the norm $\left(|\theta|_{\mathrm{L}^{2}(r \mathrm{~d} r)}^{2}+\left|\theta_{r}\right|_{\mathrm{L}^{2}(r \mathrm{~d} r)}^{2}\right)^{1 / 2}$, is isomorphic to $\mathrm{H}_{a x i}^{1}(D)$, that is the closure in $\mathrm{H}^{1}(D)$ of the set of regular axisymmetric functions.

Let $\tau_{1}, \tau_{2}, \ldots$ be a decreasing sequence of positive numbers such that $\lim \downarrow \tau_{i}=0$. We have the following existence and convergence result.

Theorem 2.1. There exists an axisymmetric function

$$
\theta \in \mathrm{L}^{\infty}\left([0,+\infty), \mathrm{H}_{a x i}^{1}(D)\right) \cap \mathrm{H}_{l o c}^{1}\left([0,+\infty), \mathrm{L}_{a x i}^{2}(D)\right)
$$

such that, up to a subsequence,

$$
\begin{array}{r}
\theta^{\tau_{i}} \underset{i \rightarrow+\infty}{\longrightarrow} \theta \quad \text { uniformly in } \mathrm{C}^{0}\left([0, T], \mathrm{L}_{a x i}^{2}(D)\right), \quad \forall T>0, \\
\theta^{\tau_{i}}(\cdot, t), \bar{\theta}^{\tau_{i}}(\cdot, t) \underset{i \rightarrow+\infty}{\longrightarrow} \theta(\cdot, t) \quad \text { weakly in } \mathrm{H}_{a x i}^{1}(D), \quad \forall t \geq 0 .
\end{array}
$$


Moreover, $\theta$ is a solution to $(5)$ in $\mathcal{D}^{\prime}((0,1) \times(0,+\infty))$, which satisfies $(6),(7)$ and the estimate:

$$
\mathcal{E}_{k}(\theta(\cdot, t))+\pi \int_{0}^{t} \int_{0}^{1}\left|\theta_{t}\right|^{2} r \mathrm{~d} r \mathrm{~d} t \quad \leq \mathcal{E}_{k}\left(\theta_{0}\right), \quad \forall t \geq 0
$$

Proof. For the axisymmetric maps, (16) implies

$$
\frac{1}{2} \int_{D \times[0,+\infty)}\left|\theta_{t}^{\tau_{i}}\right|^{2} \mathrm{~d} x \mathrm{~d} t \leq \mathcal{E}_{k}\left(\theta_{0}\right) \quad \text { and } \quad \frac{1}{2} \int_{D}\left|\nabla \bar{\theta}^{\tau_{i}}(\cdot, t)\right|^{2} \mathrm{~d} x \leq \mathcal{E}_{k}\left(\theta_{0}\right) \quad(t \geq 0)
$$

Let $T>0$. By the first inequality in $(21),\left(\theta^{\tau_{i}}\right)$ is bounded in $\mathrm{H}^{1}\left((0, T), \mathrm{L}_{a x i}^{2}(D)\right)$, which is embedded in $\mathrm{C}^{0,1 / 2}\left([0, T], \mathrm{L}_{a x i}^{2}(D)\right)$, so the sequence $\left(\theta^{\tau_{i}}\right)_{i}$ is uniformly equicontinuous in $\mathrm{C}^{0}\left([0, T], \mathrm{L}_{a x i}^{2}(D)\right)$. By convexity, the second inequality in (21) holds if we replace $\bar{\theta}^{\tau_{i}}$ by $\theta^{\tau_{i}}$, so $\left(\theta^{\tau_{i}}(t)\right)_{\tau_{i}, t \geq 0}$ is bounded in $\mathrm{H}_{a x i}^{1}(D)$, which is compactly imbedded in $\mathrm{L}_{a x i}^{2}(D)$. By Ascoli's theorem, there exist a subsequence again denoted $\left(\theta^{\tau_{i}}\right)$ and a map $\theta \in \mathrm{C}^{0}\left([0, T], \mathrm{L}_{a x i}^{2}(D)\right)$ such that $\theta^{\tau_{i}} \underset{i \rightarrow+\infty}{\longrightarrow} \theta$ uniformly in $\mathrm{C}^{0}\left([0, T], \mathrm{L}_{a x i}^{2}(D)\right)$. By diagonal extraction, we obtain a subsequence $\left(\theta^{\tau_{i}}\right)$ and $\theta \in \mathrm{C}_{l o c}^{0}\left([0,+\infty), \mathrm{L}_{a x i}^{2}(D)\right)$ such that (18) holds for every $T>0$. Notice that for this sequence, $\theta^{\tau_{i}}(\cdot, t) \underset{i \rightarrow+\infty}{\longrightarrow} \theta(\cdot, t)$ weakly in $\mathrm{H}_{a x i}^{1}(D)$ for all $t \geq 0$.

Now, having in mind the definitions of $\theta^{\tau_{i}}$ and $\bar{\theta}^{\tau_{i}},(15)$ implies

$$
\frac{1}{2} \int_{D}\left|\theta^{\tau_{i}}(\cdot, t)-\bar{\theta}^{\tau_{i}}(\cdot, t)\right|^{2} \mathrm{~d} x \leq \pi \int_{0}^{1}\left|\theta^{n+1}-\theta^{n}\right|^{2} r \mathrm{~d} r \leq \tau_{i} \mathcal{E}_{k}\left(\theta_{0}\right) \quad \forall t \in\left[n \tau_{i},(n+1) \tau_{i}\right) .
$$

Therefore, by letting $\tau_{i} \rightarrow 0$ for fixed $t, \theta^{\tau_{i}}(\cdot, t)-\bar{\theta}^{\tau_{i}}(\cdot, t) \underset{i \rightarrow+\infty}{\longrightarrow} 0$ in $\mathrm{L}_{a x i}^{2}(D)$ for all $t \geq 0$. This, together with the left inequality in (21), implies (19). By (21) again, $\left(\theta^{\tau_{i}}\right)$ is a bounded sequence in $\mathrm{H}^{1}(D \times(0, T))$ for every $T>0$. From (19),

$$
\begin{array}{ll}
\theta^{\tau_{i}} \underset{i \rightarrow+\infty}{\longrightarrow} \theta & \text { weakly in } \mathrm{H}^{1}(D \times(0, T)) \text { and a.e. in } D \times(0, T), \\
\theta_{t}^{\tau_{i}} \underset{i \rightarrow+\infty}{\longrightarrow} \theta_{t} \quad \text { weakly in } \mathrm{L}^{2}(D \times(0, T)), \quad \forall T \geq 0 .
\end{array}
$$

Thus, from (16), using in addition (19), (22) and the weak lower semi-continuity of $\mathcal{E}_{k}$, we get:

$$
\mathcal{E}_{k}(\theta(\cdot, t))+\pi \int_{0}^{t} \int_{0}^{1}\left|\theta_{t}\right|^{2} r \mathrm{~d} r \mathrm{~d} t \leq \liminf _{\tau_{i}} \mathcal{E}_{k}\left(\bar{\theta}^{\tau_{i}}(\cdot, t)\right)+\liminf _{\tau_{i}} \pi \int_{0}^{t} \int_{0}^{1}\left|\theta_{t}^{\tau}\right|^{2} r \mathrm{~d} r \mathrm{~d} t \leq \mathcal{E}_{k}\left(\theta_{0}\right)
$$

for all $t \geq 0$, and this concludes the convergence part of the theorem. The Euler equation (12) reads

$$
\theta_{t}^{\tau_{i}}=\bar{\theta}_{r r}^{\tau_{i}}+\frac{1}{r} \bar{\theta}_{r}^{\tau_{i}}+\frac{\sin 2 \bar{\theta}^{\tau_{i}}}{2 r^{2}}
$$

Using (23) and (22), we have $\theta^{\tau_{i}}, \bar{\theta}^{\tau_{i}} \rightarrow \theta$ in $\mathrm{L}_{l o c}^{2}((0,1) \times(0,+\infty))$. Letting $i \rightarrow \infty$ in (26) we get (5). 
The uniqueness of the limit $\theta$ in Theorem 2.1 is an open question in general. However, using the uniqueness of the Struwe solution [8], we can give a partial uniqueness result.

Theorem 2.2. Let $\theta_{S}$ denote the Struwe solution to (5)-(7) with initial condition $\theta_{0} \in \mathcal{S}_{\alpha}$ and let $T_{1}>0$ be the first singular time for $\theta_{S}$. Then, any limit $\theta$ obtained as in Theorem 2.1 from the semi-discrete scheme (11), with the initial condition $\theta_{0}$ and $k$ such that $\theta_{0}\left(0^{+}\right)=-\pi / 2+k \pi$, satisfies $\theta=\theta_{S}$ on $[0,1] \times\left[0, T_{1}\right)$.

In particular, if $\mathcal{E}_{k}\left(\theta_{0}\right)=\mathcal{E}\left(\theta_{0}\right)<4 \pi$, then $T_{1}=+\infty$ and the limit is unique.

Proof. First notice that for a corotational symmetric initial condition defined by $\theta_{0} \in \mathcal{S}_{\alpha}$, the Struwe solution is also by uniqueness a corotational symmetric map: it is more precisely the solution to (5)-(7) in the class (17) characterized by the fact that $\mathcal{E}(\theta(t))$ is nonincreasing. The first singular time $T_{1}$ can be defined here as the largest $T>0$ such that $\theta \in \mathrm{C}^{\infty}([0,1] \times(0, T))$.

The result of Freire stated in Lemma 2.3 below shows that $\theta=\theta_{S}$ on $\left[0, T^{\prime}\right]$ for some $T^{\prime}<T_{1}$ since $\mathcal{E}_{k}(\theta(t)) \leq \mathcal{E}_{k}\left(\theta_{0}\right)=\mathcal{E}\left(\theta_{0}\right)$ by $(20)$. The idea is to iterate the argument by showing a strong convergence on $\left[0, T^{\prime}\right]$. Define

$$
T^{+}=\sup \left\{T \in\left(0, T_{1}\right) \mid \theta=\theta_{S} \text { on }[0, T]\right\} \in\left(0, T_{1}\right]
$$

Assume by contradiction $T^{+}<T_{1}$. Then multiplying (5) by $\theta_{t}$, an integration on $[0,1] \times\left[0, T^{+}\right]$yields

$$
\mathcal{E}\left(\theta\left(\cdot, T^{+}\right)\right)+\pi \int_{0}^{T^{+}} \int_{0}^{1}\left|\theta_{t}\right|^{2} r \mathrm{~d} r \mathrm{~d} t=\mathcal{E}\left(\theta_{0}\right)
$$

The integration by parts we use here is valid because of the regularity of the Struwe solution. Together with (25), this implies

$$
\liminf _{\tau_{i}} \mathcal{E}_{k}\left(\bar{\theta}^{\tau_{i}}\left(\cdot, T^{+}\right)\right)=\mathcal{E}\left(\theta\left(\cdot, T^{+}\right)\right) \text {and } \liminf _{\tau_{i}} \pi \int_{0}^{T^{+}} \int_{0}^{1}\left|\theta_{t}^{\tau}\right|^{2} r \mathrm{~d} r \mathrm{~d} t=\pi \int_{0}^{T^{+}} \int_{0}^{1}\left|\theta_{t}\right|^{2} r \mathrm{~d} r \mathrm{~d} t
$$

Similarly to $(25),(16)$ yields: $\mathcal{E}\left(\theta_{0}\right) \leq \limsup _{\tau_{i}} \mathcal{E}_{k}\left(\bar{\theta}^{\tau_{i}}\left(\cdot, T^{+}\right)\right)+\liminf _{\tau_{i}} \pi \int_{0}^{T^{+}} \int_{0}^{1}\left|\theta_{t}^{\tau}\right|^{2} r \mathrm{~d} r \mathrm{~d} t \leq \mathcal{E}\left(\theta_{0}\right)$. Thus,

$$
\limsup _{\tau_{i}} \mathcal{E}_{k}\left(\bar{\theta}^{\tau_{i}}\left(\cdot, T^{+}\right)\right)=\liminf _{\tau_{i}} \mathcal{E}_{k}\left(\bar{\theta}^{\tau_{i}}\left(\cdot, T^{+}\right)\right)=\mathcal{E}\left(\theta\left(\cdot, T^{+}\right)\right)
$$

Now, summing (15) for $n=N^{+}, \ldots, N-1$ where $N^{+}$is defined by $N^{+} \tau \leq T^{+}<\left(N^{+}+1\right) \tau$, we get

$$
\mathcal{E}_{k}\left(\bar{\theta}^{\tau}(\cdot, N \tau)\right)+\pi \int_{N^{+} \tau}^{N \tau} \int_{0}^{1}\left|\theta_{t}^{\tau}\right|^{2} r \mathrm{~d} r \mathrm{~d} t \leq \mathcal{E}_{k}\left(\theta\left(\cdot, N^{+} \tau\right)\right)
$$

In this inequality we can pass to the limit as in $(25)$, and using $(27)$, we obtain $\mathcal{E}_{k}\left(\theta(\cdot, t) \leq \mathcal{E}\left(\theta\left(\cdot, T^{+}\right)\right.\right.$for all $t \geq T^{+}$. Thus by Lemma $2.3, \theta=\theta_{S}$ on $\left[T^{+}, T^{+}+\epsilon\right]$ for some $\epsilon>0$. This contradicts the definition of $T^{+}$and concludes the proof.

With the notations of Theorem 2.2, the uniqueness result of Freire is the following:

Lemma 2.3 (Freire [8]). Let $\theta$ be a solution to $(5),(6),(7)$ in the class (17) such that $\mathcal{E}(\theta(t)) \leq \mathcal{E}\left(\theta_{0}\right)$ for all $t \geq 0$. Then there exists $T^{\prime}$ in $\left(0, T_{1}\right)$ such that $\theta=\theta_{S}$ on $[0,1] \times\left[0, T^{\prime}\right]$. 


\section{Time AND SPACE Discretization}

For the space discretization of (11), we use piecewise linear $\left(P_{1}\right)$ continuous finite elements and, in order to deal with the singularity at 0 , we allow the nodes of the mesh to move. The unknowns of the discrete minimization problem are the values of the discrete solution at the nodes and the position of the nodes. Similarly to the steady-state case, the discrete solution minimizes a relaxed discrete functional, and its graph may have a vertical part at $r=0$.

Let $M$ be a positive integer and let $h>1 / M$. The (non-empty) set of admissible subdivisions is

$$
D^{M, h}:=\left\{\left(r_{i}\right)_{0 \leq i \leq M} \in \mathbb{R}^{M+1}, r_{0}=0<r_{1}<\cdots<r_{M}=1 \text { and } \max _{1 \leq i \leq M}\left|r_{i}-r_{i-1}\right| \leq h\right\}
$$

In the following, we assume that $h=h(M)>1 / M$ is a function of $M$ and we omit the index $M$. The space of moving finite elements is

$$
\begin{gathered}
H_{k, \alpha}^{h}:=\left\{\theta^{h} \in C^{0}([0,1]), \theta^{h}(0)=-\frac{\pi}{2}+k \pi, \theta^{h}(1)=\alpha, \exists\left(r_{i}\right)_{0 \leq i \leq M} \in D^{h}\right. \text { such that } \\
\left.\theta^{h} \text { is affine on }\left[r_{i}, r_{i+1}\right] \forall i \in\{1, \ldots, M\}\right\} .
\end{gathered}
$$

Let $k \in \mathbb{Z}$ and $\tau>0$ be fixed. For an initial data $\theta_{0}^{h} \in H_{k, \alpha}^{h}$, the full discretized scheme is:

$$
\theta_{n+1}^{h} \text { "minimizes" } \mathcal{F}_{k, n}^{h}\left(\phi^{h}\right):=\mathcal{E}\left(\phi^{h}\right)+\frac{\pi}{\tau} \int_{0}^{1}\left|\phi^{h}-\theta_{n}^{h}\right|^{2} r \mathrm{~d} r \text { among } \phi^{h} \in H_{k, \alpha}^{h}
$$

However, a minimizer for problem (30) may not exist, because $H_{k, \alpha}^{h}$ is not closed. As in the steady-state case [1], we use the following construction (compare with (10)):

Lemma 3.1. Let $\Omega \subset \mathbb{R}^{m}$ and let $\mathcal{F}: \Omega \rightarrow[0,+\infty)$ be continuous on $\Omega$. Then, $\overline{\mathcal{F}}: \bar{\Omega} \rightarrow[0,+\infty]$, defined on the closure of $\Omega$ in $\mathbb{R}^{m}$ by

$$
\overline{\mathcal{F}}(x):=\inf \left\{\liminf _{p} \mathcal{F}\left(x_{p}\right) \mid x_{p} \in \Omega, x_{p} \rightarrow x\right\} \quad(x \in \bar{\Omega})
$$

is an extension of $\mathcal{F}$ which is lower semi-continuous (l.s.c.) on $\bar{\Omega}$.

A function $\theta^{h} \in H_{k, \alpha}^{h}$ is uniquely defined by the sequence $\left(r_{i}, \theta_{i}\right)_{0 \leq i \leq M}$, where $\left(r_{i}\right)_{0 \leq i \leq M}$ are the nodes and $\theta_{i}=\theta^{h}\left(r_{i}\right)$ the values at the nodes. With this identification,

$$
H_{k, \alpha}^{h} \simeq \Omega_{k, \alpha}^{h}:=D^{h} \times\left(\{-\pi / 2+k \pi\} \times \mathbb{R}^{M-1} \times\{\alpha\}\right) \subset \mathbb{R}^{2 M+2}
$$


and the functional $\mathcal{F}_{k, n}^{h}$ is clearly continuous on $\Omega_{k, \alpha}^{h} \subset \mathbb{R}^{2 M+2}$. Using Lemma 3.1 with $\Omega=\Omega_{k, \alpha}^{h} \subset \mathbb{R}^{2 M+2}$ and $\mathcal{F}=\mathcal{F}_{k, n}^{h}$, we obtain the extended minimization problem:

$$
\bar{\theta}_{n+1}^{h} \simeq\left(r_{i}^{n+1}, \theta_{i}^{n+1}\right)_{0 \leq i \leq M} \in \overline{\Omega_{k, \alpha}^{h}} \text { minimizes } \overline{\mathcal{F}_{k, n}^{h}}\left(\bar{\phi}^{h}\right) \text { among } \bar{\phi}^{h} \in \overline{\Omega_{k, \alpha}^{h}},
$$

where $\bar{\theta}_{0}^{h}$ is chosen in $\overline{\Omega_{k, \alpha}^{h}}$. The following estimate:

$$
\mathcal{F}_{k, n}^{h}\left(\theta^{h}\right) \geq \mathcal{E}\left(\phi^{h}\right) \geq \pi \sum_{i=0}^{M-1} \frac{\left(\theta_{i+1}-\theta_{i}\right)^{2}}{2}\left(\frac{r_{i+1}+r_{i}}{r_{i+1}-r_{i}}\right) \geq \pi \sum_{i=0}^{M-1} \frac{\left(\theta_{i+1}-\theta_{i}\right)^{2}}{2}
$$

extends to $\overline{\mathcal{F}_{k, n}^{h}}$, by continuity of the right-hand side. Thus, a minimizing sequence for problem (32) is bounded; it converges, up to a subsequence, to some $\bar{\theta}_{n+1}^{h} \in \overline{\Omega_{k, \alpha}^{h}}$, which is a minimizer since $\overline{\mathcal{F}_{k, n}^{h}}$ is l.s.c. In particular, problem (32) has a solution.

This discrete solution has the same structure as in the steady-state case [1].

More precisely, let $\bar{\theta}^{h}=\left(\bar{r}_{i}, \bar{\theta}_{i}\right)_{0 \leq i \leq M} \in \overline{\Omega_{k, \alpha}^{h}}$ such that $\overline{\mathcal{F}_{k, n}^{h}}\left(\bar{\theta}^{h}\right)<+\infty$. By definition, there exists a sequence $\left(\theta^{h, p}\right)_{p}=\left(\left(r_{i}^{p}, \theta_{i}^{p}\right)_{0 \leq i \leq M}\right)_{p}$ in $H_{k, \alpha}^{h} \simeq \Omega_{k, \alpha}^{h}$ such that $\sup _{p} \mathcal{E}\left(\theta^{h, p}\right)<\infty$ and $\left(\left(r_{i}^{p}, \theta_{i}^{p}\right)_{0 \leq i \leq M}\right)_{p}$ converges to $\left(\bar{r}_{i}, \bar{\theta}_{i}\right)_{0 \leq i \leq M}$ in $\mathbb{R}^{2 M+2}$. Necessarily, $0=\bar{r}_{0} \leq \bar{r}_{1} \leq \cdots \leq \bar{r}_{M}=1$, and from estimate (33), for every $i \in\{0, \ldots, M-1\}$ such that $\bar{r}_{i}=\bar{r}_{i+1}>0, \bar{\theta}_{i}=\bar{\theta}_{i+1}$. However, we may have $\bar{r}_{i}=\bar{r}_{i+1}=0$ and simultaneously, $\bar{\theta}_{i} \neq \bar{\theta}_{i+1}$ : this corresponds to a vertical part at $r=0$ in the graph of $\bar{\theta}$. On the other hand, the sequence $\left(\theta^{h, p}\right)_{p}$ is bounded in $\mathrm{H}_{a x i}^{1}(D)$, so it converges, up to a subsequence, weakly in $\mathrm{H}_{a x i}^{1}(D)$ to some $\theta^{h}$, with $\mathcal{E}_{k}\left(\theta^{h}\right) \leq \liminf _{p} \mathcal{E}\left(\theta^{h, p}\right)<\infty$. By strong convergence, $\theta^{h} \in \mathcal{S}_{\alpha}$ is a continuous piecewise linear function which can be identified to $\left(\bar{r}_{i}, \bar{\theta}_{i}\right)_{i_{0} \leq i \leq M}$, where $i_{0}:=\max \left\{i: \bar{r}_{i}=0\right\}: \theta^{h}$ is the regular part of $\bar{\theta}^{h}$. The discrete energy of the vertical part is equal to

$$
\overline{\mathcal{F}_{k, n}^{h}}\left(\bar{\theta}^{h}\right)-\left(\mathcal{E}\left(\theta^{h}\right)+\frac{\pi}{\tau} \int_{0}^{1}\left|\theta^{h}-\theta_{n}^{h}\right|^{2} r \mathrm{~d} r\right) \geq \mathcal{E}_{k}\left(\theta^{h}\right)-\mathcal{E}\left(\theta^{h}\right)=4\left|\theta^{h}\left(0^{+}\right)-k \pi+\frac{\pi}{2}\right|
$$

In other words, the scheme (32) is a space discretization of (11) which includes the vertical part. We have the following convergence result, where $\theta_{n}^{h} \in \mathcal{S}_{\alpha}$ denotes the regular part of $\bar{\theta}_{n}^{h} \in \overline{\Omega_{k, \alpha}^{h}}$.

Theorem 3.2. Let $k \in \mathbb{Z}, \tau>0$ and $\theta_{0} \in \mathcal{S}_{\alpha}$. Consider a sequence of initial values $\bar{\theta}_{0}^{h} \in \overline{\Omega_{k, \alpha}^{h}}$ such that $\theta_{0}^{h} \underset{h \rightarrow 0}{\longrightarrow} \theta_{0}$ in $\mathrm{L}_{a x i}^{2}(D)$. For every $h>0$, let us call $\bar{\theta}_{1}^{h}, \bar{\theta}_{2}^{h}, \ldots$ a sequence constructed by (32) with the initial value $\bar{\theta}_{0}^{h}$. Then, up to a subsequence,

$$
\theta_{n}^{h} \underset{h \rightarrow 0}{\longrightarrow} \theta_{n} \text { in } \mathrm{L}_{a x i}^{2}(D) \quad(n \in \mathbb{N})
$$

where $\theta_{1}, \theta_{2}, \ldots$ is a sequence satisfying (11) with the initial value $\theta_{0}$. Moreover,

$$
\overline{\mathcal{F}_{k, n-1}^{h}}\left(\bar{\theta}_{n}^{h}\right) \underset{h \rightarrow 0}{\longrightarrow} \mathcal{E}_{k}\left(\theta_{n}\right)+\frac{\pi}{\tau} \int_{0}^{1}\left|\theta_{n}-\theta_{n-1}\right|^{2} r d r \quad\left(n \in \mathbb{N}^{*}\right) .
$$


Proof. By recursivity, we only have to prove the claim for $n=1$. Let $\phi \in \mathcal{H}_{k, \alpha}$, and let $I^{h} \phi$ denote the continuous piecewise linear interpolate of $\phi$ on the uniform subdivision $r_{i}=i h, 0 \leq i \leq M$ (recall that $\left.h=h(M)>1 / M\right)$. Then, $I^{h} \phi \in \mathcal{H}_{k, \alpha}^{h}$, so

$$
\mathcal{E}_{k}\left(\theta_{1}^{h}\right)+\frac{\pi}{\tau} \int_{0}^{1}\left|\theta_{1}^{h}-\theta_{0}^{h}\right|^{2} r d r \leq \overline{\mathcal{F}_{k, 0}^{h}}\left(\bar{\theta}_{1}^{h}\right) \leq \mathcal{E}\left(I^{h} \phi\right)+\frac{\pi}{\tau} \int_{0}^{1}\left|I^{h} \phi-\theta_{0}^{h}\right|^{2} r \mathrm{~d} r
$$

First, we let $\phi$ be the affine function on $[0,1]$ such that $\phi(0)=-\pi / 2+k \pi$ and $\phi(1)=\alpha$. For this choice, $\phi=I^{h} \phi$, so the right-hand side of (34) is clearly bounded as $h \rightarrow 0$. Hence, the sequence $\left(\theta_{1}^{h}\right)_{h}$ is bounded in $\mathrm{H}_{a x i}^{1}(D)$; it converges, up to a subsequence, strongly in $\mathrm{L}_{a x i}^{2}(D)$ to some $\theta_{1}$. Passing to the limit in the left inequality of (34), and using the lower semi-continuity of $\mathcal{E}_{k}$ in $\mathcal{S}_{\alpha}$, we get

$$
\mathcal{E}_{k}\left(\theta_{1}\right)+\frac{\pi}{\tau} \int_{0}^{1}\left|\theta_{1}-\theta_{0}\right|^{2} r \mathrm{~d} r \leq \liminf _{h} \overline{\mathcal{F}_{k, 0}^{h}}\left(\bar{\theta}_{1}^{h}\right)
$$

Now let $\phi$ be any function in $\mathcal{H}_{k, \alpha}^{h}$. By regularity of $\phi$ (see [11] for details),

$$
\mathcal{E}\left(I^{h} \phi\right) \underset{h \rightarrow 0}{\longrightarrow} \mathcal{E}\left(I^{h} \phi\right) \text { and } I^{h} \phi \underset{h \rightarrow 0}{\longrightarrow} \phi \text { strongly in } \mathrm{L}_{a x i}^{2}(D) .
$$

Thus, passing to the limit in the right inequality of (34), we obtain

$$
\underset{h}{\limsup } \overline{\mathcal{F}_{k, 0}^{h}}\left(\bar{\theta}_{1}^{h}\right) \leq \mathcal{E}(\phi)+\frac{\pi}{\tau} \int_{0}^{1}\left|\phi-\theta_{0}\right|^{2} r \mathrm{~d} r
$$

This is true for every $\phi \in \mathcal{H}_{\alpha, k}$, so together with (10) and (35), it shows that $\theta_{1}$ is a solution to (11) for $n=0$, and concludes the proof.

\section{Computational Considerations}

For the computation of (32), there are two difficulties: first, the energy of regular $P_{1}$ functions (without vertical part), which cannot be exact because of the cosine term, and second, the (relaxed) discrete energy of vertical parts. These difficulties are solved as in the steady-state case [1]: we use a discretization of the energy by the Gaussian quadrature with two nodes, and a change of variable on the mesh; it happens that the l.s.c. extension of the discrete energy, which we define as previously by Lemma 3.1, is smooth with respect to the new unknowns, even at vertical parts. In other words, we discretize problem (30) by a quadrature formula, and we consider the corresponding extended minimization problem (in the new unknowns), obtained by Lemma 3.1. It seems reasonable to expect that the minimizer for this problem is close in $\mathbb{R}^{2 M+2}$ to the minimizer of problem (32) and that their discrete energies are close. With this approach, we also avoid working with the "jumping" unknown $\theta^{h}\left(0^{+}\right)$.

For $\theta^{h} \simeq\left(r_{i}, \theta_{i}\right)_{0 \leq i \leq M} \in \mathcal{H}_{k, \alpha}^{h}$, the approximation of $\mathcal{E}\left(\theta^{h}\right)$ by the Gaussian quadrature with two nodes $c_{1}=1 / 2-\sqrt{3} / 6$ and $c_{2}=1 / 2+\sqrt{3} / 6$ yields

$$
E_{G}^{h}\left(\theta^{h}\right):=\pi \sum_{i=0}^{M-1} e_{G}^{h}\left(t_{i}, \theta_{i}, \theta_{i+1}\right)
$$

where, for every $0 \leq i \leq M-1, t_{i}=r_{i} / r_{i+1}$ and

$$
e_{G}^{h}\left(t_{i}, \theta_{i}, \theta_{i+1}\right):=\left[\frac{\cos ^{2}\left(c_{2} \theta+c_{1} \theta_{i+1}\right)}{c_{2} t_{i}+c_{1}}+\frac{\cos ^{2}\left(c_{1} \theta+c_{2} \theta_{i+1}\right)}{c_{1} t_{i}+c_{2}}\right]\left(\frac{1-t_{i}}{2}\right)+\frac{\left(\theta_{i+1}-\theta\right)^{2}}{2}\left(\frac{1+t_{i}}{1-t_{i}}\right) .
$$


The fact that $e_{G}^{h}$ depends only on the ratio $t_{i}=r_{i} / r_{i+1}$ is related to the conforming invariance of the Dirichlet energy in two dimensions. For the computation, we use this variable $t_{i}$, because $e_{G}^{h}$ is smooth at $t_{i}=0$, and this corresponds to a vertical part. Seeing $D^{h}(28)$ as a subset of $\mathbb{R}^{M-1}$ (because $r_{0}=0$ and $r_{M}=1$ ), the change of variable $\Phi^{h}: D^{h} \ni\left(r_{1}, \ldots, r_{M-1}\right) \mapsto\left(t_{1}, \ldots, t_{M-1}\right) \in(0,1)^{M-1}$ is a smooth diffeomorphism from $D^{h}$ onto $\Phi^{h}\left(D^{h}\right)$. For every $t=\left(t_{1}, \ldots, t_{M-1}\right) \in \Phi^{h}\left(D^{h}\right)$, the pre-image $r=\left(r_{1}, \ldots, r_{M-1}\right)$ can be computed by

$$
\left\{\begin{array}{l}
r_{M}=1, \\
r_{i}=t_{i} r_{i+1} \text { for } i=M-1, \ldots, 0 .
\end{array}\right.
$$

We also see $\Omega_{k, \alpha}^{h}(31)$ as $D^{h} \times \mathbb{R}^{M-1}$ because the values $\theta_{0}=-\pi / 2+k \pi$ and $\theta_{M}=\alpha$ are fixed. For every $\Phi^{h} \in$ $\Omega_{k, \alpha}^{h} \simeq D^{h} \times \mathbb{R}^{M-1}$, let $F_{k, n}^{h}\left(\phi^{h}\right):=E_{G}^{h}\left(\phi^{h}\right)+\frac{\pi}{\tau} \int_{0}^{1}\left|\phi^{h}-\theta_{n}^{h}\right|^{2} r \mathrm{~d} r$. Using Lemma 3.1 with $\Omega=\Phi^{h}\left(D^{h}\right) \times \mathbb{R}^{M-1}$ and $\mathcal{F}\left(\phi^{h}\right)=F_{k, n}^{h}\left(\phi^{h}\right)$, where $F_{k, n}^{h}$ is seen as a function of $\left(t_{i}, \theta_{i}\right)_{1 \leq i \leq M-1}$ through $\Phi^{h}$, we have coded for every $n$ the following minimization problem:

$$
\bar{\theta}_{n+1}^{h} \simeq\left(t_{i}^{n+1}, \theta_{i}^{n+1}\right)_{1 \leq i \leq M-1} \in \overline{\Phi^{h}\left(D^{h}\right)} \times \mathbb{R}^{M-1} \text { minimizes } \overline{F_{k, n}^{h}} \text { in } \overline{\Phi^{h}\left(D^{h}\right)} \times \mathbb{R}^{M-1},
$$

where $\bar{\theta}_{0}^{h}$ is chosen in $\overline{\Phi^{h}\left(D^{h}\right)} \times \mathbb{R}^{M-1}$. By the same arguments as in Section 3, this problem has a solution.

Remark 4.1. The meaning of $\bar{\theta}_{n}^{h}$ in variables $\left(r_{i}, \theta_{i}\right)_{1 \leq i \leq M-1}$ is the following: apply Lemma 3.1 with $\Omega=\Omega_{k, \alpha}^{h}$ and $\mathcal{F}\left(\phi^{h}\right)=F_{k, n}^{h}\left(\phi^{h}\right)$, and consider for every $n$ the following approximation of problem (32):

$$
\bar{\theta}_{n+1}^{h} \simeq\left(r_{i}^{n+1}, \theta_{i}^{n+1}\right)_{0 \leq i \leq M} \in \overline{\Omega_{k, \alpha}^{h}} \operatorname{minimizes} \overline{F_{k, n}^{h}}\left(\bar{\phi}^{h}\right) \text { among } \bar{\phi}^{h} \in \overline{\Omega_{k, \alpha}^{h}} \text {. }
$$

The infimum for $F_{k, n}^{h}$ in $\Phi^{h}\left(D^{h}\right) \times \mathbb{R}^{M-1}$ is the same as in $\Omega_{k, \alpha}^{h}$, by $\Phi^{h}$. By density, the infimum for problem (40) is the same as the infimum for problem (39). Moreover, by the same arguments as in Section 3, problem (40)

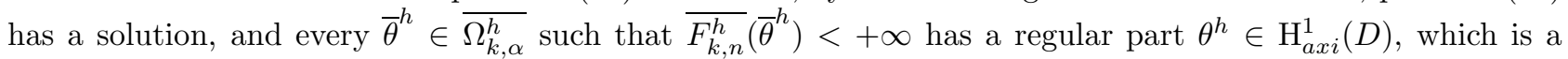
continuous piecewise linear function, and a vertical part at $r=0$. The constraint $\theta_{0}=-\pi / 2+k \pi$ is still true, but we may not have $\theta^{h}\left(0^{+}\right) \in \pi / 2+\pi \mathbb{Z}$ : the elements are nonconforming. The numerical simulations in section 6 show that $\theta^{h}\left(0^{+}\right) \notin \pi / 2+\pi \mathbb{Z}$ indeed, but $\theta^{h}\left(0^{+}\right) \rightarrow \pi / 2+l \pi$ for some $l \in \mathbb{Z}$ as $h$ decreases.

The minimization algorithm for (39) is a Fletcher-Reeves conjugate gradient [12]. The line search is done with a dichotomie. Working with $\left(t_{i}\right)_{i} \in \overline{\Phi^{h}\left(D^{h}\right)}$ instead of $\Phi^{h}\left(D^{h}\right) \subset(0,1)^{M-1}$ implies that the values $t_{i}=0$ and $t_{i}=1$ are allowed. We deal with the affine inequality constraints $t_{i} \in[0,1]$ as in a projected gradient method (see [1] for details). The activation of the constraint $t_{i}=0$ for some $i>0$, which corresponds to a vertical part, does happen exactly: we call it a numerical bubbling. In this case, if $i_{0}:=\max \left\{i: t_{i}=0\right\}$, then $\theta^{h}\left(0^{+}\right)=\theta_{i_{0}}$ and the corresponding vertical segment is $\left[(0,-\pi / 2+k \pi),\left(0, \theta_{i_{0}}\right)\right]$. The activation of the constraint $t_{i}=1$ also happens: in this case, the points $\left(t_{i}, \theta_{i}\right)$ and $\left(t_{i+1}, \theta_{i+1}\right)$ are equal from (37). This tangling difficulty is well-known in the context of moving-mesh methods. It can be cured by the introduction of internodal forces [5]. Here, such methods are not necessary. We introduce a small parameter $\epsilon_{t}>0$ (typically $\left.\epsilon_{t} \in[0.005,0.01]\right)$, and whenever $t_{i}>1-\epsilon_{t}$, we substract one point (the right one or the left one, whether we are close to 0 or 1 respectively). Concerning the line search, a maximal step-size $\lambda_{\max }$ is given by the conditions $0 \leq t_{i}+\lambda_{\max } \mathrm{d} t_{i} \leq 1-\epsilon_{t} / 2$, where $\mathrm{d} t_{i}$ is the coordinate of the descent direction corresponding to $t_{i}$.

As in the stationary case, the minimization algorithm has two phases, as shown in Figure 3. During the first phase, the energy decreases quickly and we obtain within a relatively few number of iterations (typically $2 M$, i.e. of the same order than the number of unknowns) the graph of the solution. During the second phase, the gradient of the energy converges slowly to 0 with an oscillation phenomenon due to the singularity of the Hessian of the function with respect to the $2(M-1)$ variables $\left(t_{i}, \theta_{i}\right)$ (the hessian is close to a singular matrix of rank $M-1$ ). The second phase corresponds to the points moving tangently along the graph (see [1]) and possibly tangling. Typically, if a portion of the current solution $\theta^{n}$ is close to a segment, then the minimization 


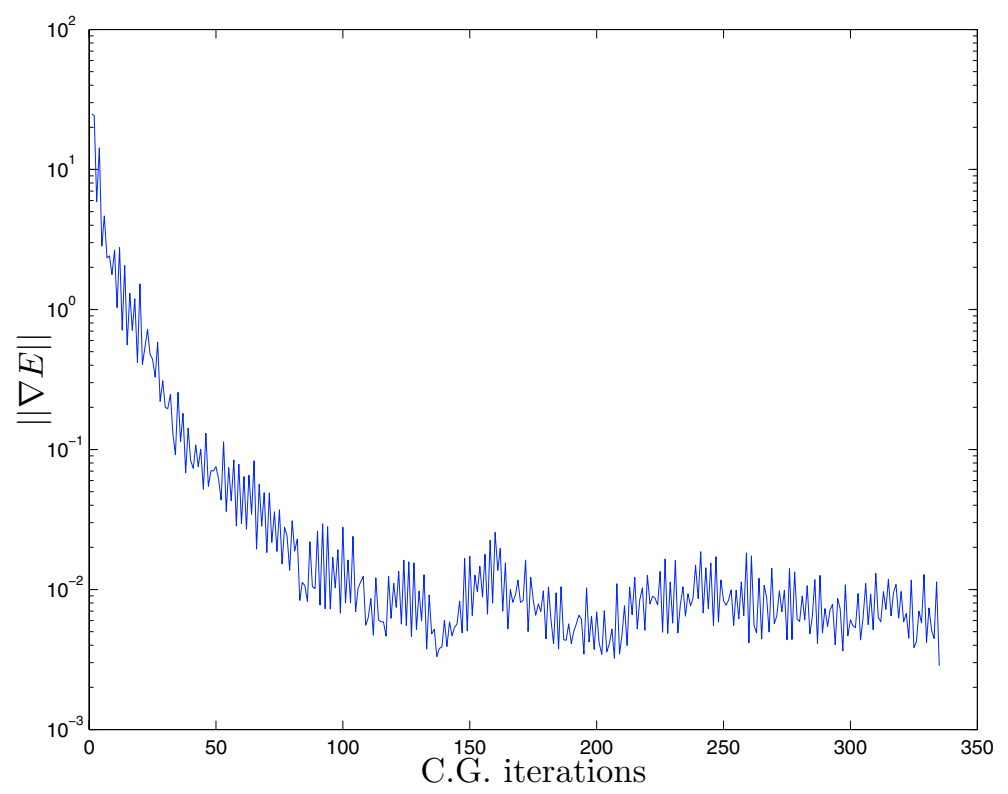

Figure 3. Convergence of the conjugate gradient for $M=80$ points (bubbling case, $\tau=0.005$ ).

algorithm is badly conditioned [1] and the points tend to flee the region of the segment and disappear; starting with 20 points we would end up after a few time iterations with a small number of points.

Thus, we stop the minimization at the end of the first phase, by limiting the number of iterations to $3 M$. This is a way to prevent undesired tangential movements of the points along the graph, and it allows the condition $\left|r_{i+1}-r_{i}\right| \leq h$ in definition (28) to be satisfied ( $h$ is not fixed a priori); this guarantees the consistency of the discretization.

\section{Numerical CONVERGENCE}

In this section, we are interested in numerical error estimations concerning the space discretization.

Let $\left(\theta_{n}^{h}\right)_{n}$ denote (the regular part of) a sequence obtained by the full discretized scheme (40) for a movingmesh or for a fixed mesh (in the latter case, $D^{h}$ is replaced by a single mesh in $D^{h}$ ). We shall denote $\theta^{\tau, h}$ the (time) continuous piecewise linear interpolate associated to the sequence $\left(\theta_{n}^{h}\right)_{n}$ as in (13). We first introduce an error estimator concerning the space discretization, inspired by Theorem 2.1:

$$
e_{\tau}(h):=\sup _{t \in[0, T]}\left\|\theta^{\tau, h}(t)-\theta^{\tau, 0^{+}}(t)\right\|_{L_{a x i}^{2}(D)}
$$

where we choose the final time $T=0.4$ large enough so that $\theta^{\tau, h}(T)$ is close to the stationary solution. Since the true solution $\theta^{\tau, 0^{+}}=\theta^{\tau}$ obtained (in part) from Theorem 3.2 by letting $h \rightarrow 0$ is unknown, we used instead the solution $\theta^{\tau, h}$ on a finer space-mesh.

We first computed $e_{\tau}(h)$ for a regular case and for fixed uniform meshes with step-size $h(h=10,20,40,80$ and 160) and for fixed $\tau(\tau=0.04,0.02$ and 0.01). We had set $\alpha=-\pi / 4, k=0$, and the starting data was given by $\theta_{0}(r)=\sin (2 \pi r)+(\alpha+\pi / 2) r-\pi / 2$ for all $r \in[0,1]$, or more precisely, its $P_{1}$ interpolate $I^{h} \theta_{0}$ on the uniform mesh. The fine space-mesh for the computation of $\theta^{\tau, 0^{+}}$was fixed and uniform with step-size $h=1 / 1280$. 


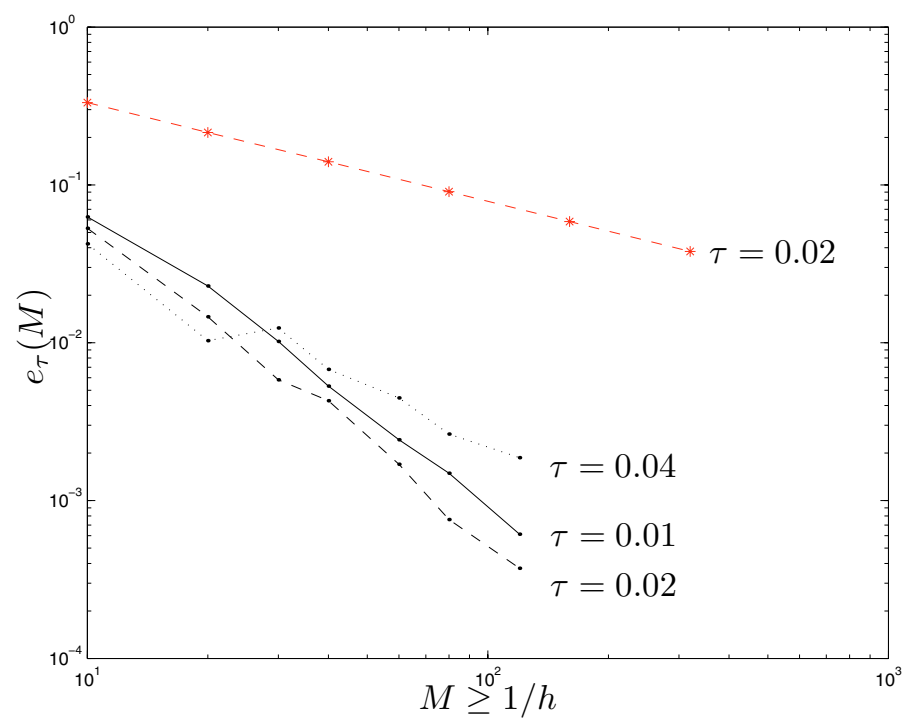

FigurE 4. Error of the space discretization for fixed uniform meshes (upper line) and moving meshes (other lines): bubbling case.

We found that, in agreement with Theorem 3.2, the error decreases as $h$ decreases. More precisely, $e_{\tau}(h) \approx$ $C(\tau) h^{\gamma}$ with $\gamma=-2.00$ up to 0.01 ; the constant $C(\tau)$ is very stable as $\tau$ decreases. We also found that the error at the final time $T$ satisfies $\left\|\theta^{\tau, h}(T)-\theta^{\tau, 0^{+}}(T)\right\|_{\mathrm{L}_{a x i}^{2}(D)}=C(\tau, T) h^{2}$, with a smaller constant $C(\tau, T)$. The exponent $\gamma=2$ is also the same for the steady-state case [1].

The interpretation of $\gamma=2$ is that the error on the space discretization is of the same order as the interpolation error. That is, for any regular $\theta \in \mathcal{H}_{k, \alpha}$, if $I^{h}(\theta)$ denotes the $P_{1}$ interpolate of $\theta$ on a uniform mesh with stepsize $h$, we have $\left\|I^{h}(\theta)-\theta\right\|_{\mathrm{L}_{a x i}^{2}(D)} \leq C h^{2}$ for some constant $C$ depending on $\theta$. Notice that we have such an interpolation error on the initial condition in our computations, since $\theta_{0}^{h}=I^{h}\left(\theta_{0}\right)$.

Figure 4 represents the error $e_{\tau}(h)$ of the space discretization as a function of $h$ for fixed uniform meshes with step-size $h=1 / M$ (upper line) and for moving meshes with $M>1 / h$ nodes (other lines). It concerns a bubbling situation with $\alpha=-\pi / 4, k=2$ and $\theta_{0}(r)=(\alpha-3 \pi / 2) \sin (\pi r / 2)+3 \pi / 2$. The fine space-mesh for the computation of $\theta^{\tau, 0^{+}}$is a fixed mesh with $M=2000$ nodes refined near 0 ; it was obtained by the movingmesh algorithm applied to the steady-state case [1]; its regular part contains approximately 800 nodes. For the moving-mesh, we have represented $e_{\tau}(h)$ for $\tau=0.04, \tau=0.02$ and $\tau=0.01$ respectively; for the uniform mesh, only the error $e_{\tau}(h)$ corresponding to $\tau=0.02$ has been represented, because the lines corresponding to $\tau=0.01,0.02$ and 0.04 respectively almost merge, as in the regular case.

In agreement with Theorem 3.2, the error decreases as $h \rightarrow 0$. For the fixed mesh, we find that $e_{\tau}(h)=C(\tau) h^{\gamma}$ with $\gamma=0.63$ up to 0.01 ; in comparison with the regular case, this exponent is smaller because of the loss of regularity of the solution. For the moving mesh the convergence is better, although it is not as stable as for the fixed mesh (oscillations in the convergence graph). In comparison with the moving mesh in the stationary case [1], where the convergence graph in logarithmic scale was a line, this unstability is due to the $L^{2}$ term in the functional $F_{k, n}^{h}$, which increases the interdependency of the nodes. However, we find that in average, $e_{\tau}(1 / M) \approx C(\tau) M^{-\gamma}$, where the exponent $\gamma$, obtained by a least square method, is $1.9,2.0$ and 1.4 for $\tau=0.01$, 0.02 and 0.04 respectively. For $\tau=0.01$ and 0.02 , this exponent $\gamma$ is very close to the one found previously in the regular case with fixed uniform meshes $(\gamma=2)$ and also for the moving mesh in the stationary problem [1]. This can be interpreted as the fact that the moving mesh, which is a mesh adapted to the graph of the solution, does not "see" the singularity. 


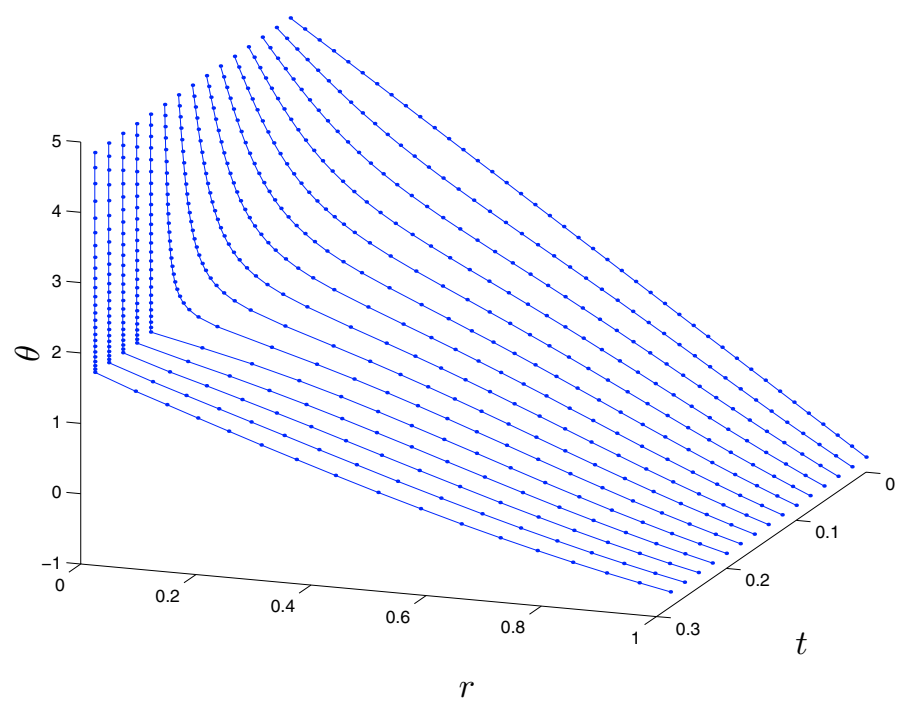

FiguRE 5. Bubbling phenomenon.

\section{NumericAl RESUlts}

Figure 5 represents a bubbling case computed for $\tau=0.02$ by the moving-mesh algorithm (40). Here, $\alpha=-\pi / 4, k=2$, the initial data is the affine function $\theta_{0}(r)=(\alpha-3 \pi / 2) r+3 \pi / 2$ for all $r \in[0,1]$ and the initial mesh is uniform with step-size $h=1 / 40$. We have represented the computed $\theta^{\tau, h}(r, t)$ as a function of $r$ in coordinates $(t, r, \theta)$ for every $t=n \tau$ with $n=0, \ldots, 14$.

We see the concentration at $r=0$ and the preservation of the degree (i.e. of the boundary condition $3 \pi / 2$ at $r=0$ ) for the graph of the solution. The numerical bubbling time is $t=0.20$ up to $\tau=0.02$. The distribution of the points along the singularity is very close to the distribution obtained in the stationary case with the Gauss formula [1]; it corresponds heuristically to a uniform distribution of the discrete energy. Because of the consistency error, the lowest $\theta_{i_{0}}$ on the singularity is not exactly $\pi / 2$, as it should be if we used the exact energy $\mathcal{E}$ instead of $E_{G}^{h}$. At bubbling time, it is equal to $\theta_{i_{0}}=\pi / 2+0.0435$. This is a little worse than the consistency error obtained in the stationary case, because of the competition of the $\mathrm{L}^{2}$ term. However the distribution of the $\theta_{i}$ along the singularity improves slightly after bubbling time: at $t=0.28$ for instance we have $\theta_{i_{0}}=\pi / 2+0.0190$. and for $t>0.40$, when the discrete solution is almost stationary, $\theta_{i_{0}}=\pi / 2+0.0157$. The final solution obtained for large $t$ is very close to the solution obtained in the stationary case [1]. In this example there is no tangling phenomenon: the number of nodes for large $t$ is the same as for $t=0$, that is 41 .

Figure 6 shows a bubbling and reverse bubbling phenomenon computed by the moving-mesh algorithm. We have used $\alpha=-\pi / 4, k=0$ and the initial data $\theta_{0}$ is the continuous piecewise linear function, relative to the subdivision $0<1 / 6<2 / 3<1$ of [0,1], defined by its values at $r=0,1 / 6,2 / 3$ and 1 which are $-\pi / 2,4,3$ and $\alpha$ respectively. The 41 nodes of the initial mesh are distributed in such a way that the interval $[0,1 / 6]$ is uniformly divided into 13 segments, the interval [1/6,2/3] into 13 segments and [2/3, 1] into 14 segments. Here, $\tau=0.02$ and the computed solution is represented at times $t=n \tau$ with $n=0, \ldots, 19$.

As previously, we see the preservation of the degree (here the boundary condition at $r=0$ is $-\pi / 2$ ). The bubbling occurs at the second time iteration $t=0.04$, with a concentration at $r=0$ and a distribution of the points along the singularity similar to the previous case. Notice that, although we deal with $P_{1}$ elements, the 

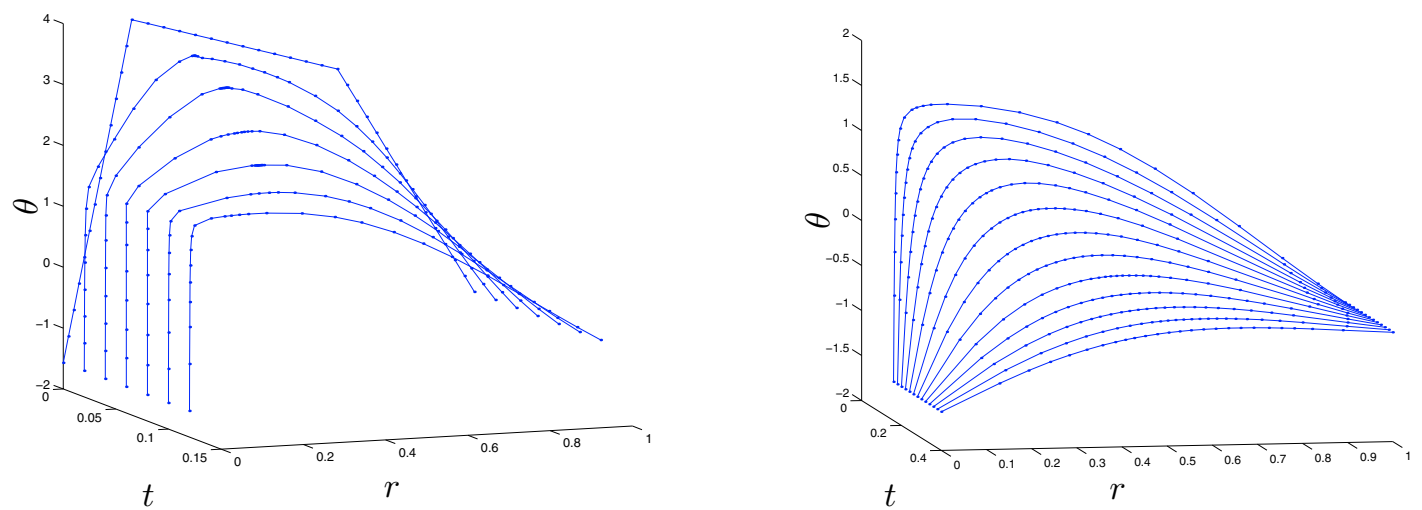

FiguRE 6. Reverse bubbling phenomenon $-t \in[0,0.12]$ and $t \in[0.14,0.38]$.

solution at the first time iteration $t=0.02$ looks "smoother" than the initial condition $\theta_{0}$ : this correspond to a smoothing phenomenon well-known for the heat equation in general. The energy stays concentrated for $t \in[0.04,0.08]$ and the debubbling occurs at $t=0.10$. The solution for $t \geq 0.10$ is smooth and converges to the stationary harmonic map $\theta^{\infty}$ of degree $k=0$ corresponding to the boundary values $\theta^{\infty}(0)=-\pi / 2$ and $\theta^{\infty}(1)=-\pi / 4$.

In Figure 6, we see an example of the tangling phenomenon related to the moving-mesh approach. Starting with 41 nodes, we end up with 26 nodes. The nodes tangle between the time iteration 3 to 6 . At $t=0.02$ we still have 41 nodes, at $t=0.04$ we have lost 3 nodes where $\theta^{\tau, h}(\cdot, t)$ reaches its maximum; at $t=0.06$ we lose 5 more nodes at the same place; at $t=0.08$ we still have 33 nodes but at $t=0.10$ the number of nodes is 26 , and this number will be constant everafter. The tangling phenomenon here is actually rather welcome in our geometrical point of view, since the length of the graph of the solution $\theta^{\tau, h}(\cdot, t)$ decreases in the same time as the number of nodes decreases; hence the ratio of these two quantities, which is an estimator of the error concerning the space discretization, stays close to the initial ratio.

\section{REFERENCES}

[1] F. Alouges and M. Pierre, Mesh optimization for singular axisymmetric harmonic maps from the disc into the sphere. Numer. Math. To appear.

[2] F. Bethuel, J.-M. Coron, J.-M. Ghidaglia and A. Soyeur, Heat flows and relaxed energies for harmonic maps, in Nonlinear diffusion equations and their equilibrium states, 3 (Gregynog, 1989), Birkhäuser Boston, Boston, MA. Progr. Nonlinear Differential Equations Appl. 7 (1992) 99-109.

[3] M. Bertsch, R. Dal Passo and R. van der Hout, Nonuniqueness for the heat flow of harmonic maps on the disk. Arch. Rational Mech. Anal. 161 (2002) 93-112.

[4] H. Brezis and J.-M. Coron, Large solutions for harmonic maps in two dimensions. Comm. Math. Phys. 92 (1983) $203-215$.

[5] N. Carlson and K. Miller, Design and application of a gradient-weighted moving finite element code. I. In one dimension. SIAM J. Sci. Comput. 19 (1998) 728-765.

[6] K.-C. Chang, Heat flow and boundary value problem for harmonic maps. Ann. Inst. H. Poincaré Anal. Non Linéaire 6 (1989) 363-395.

[7] J. Eells and J. Sampson, Harmonic mappings of Riemannian manifolds. Amer. J. Math. 86 (1964) 109-160.

[8] A. Freire, Uniqueness for the harmonic map flow from surfaces to general targets. Comment. Math. Helv. 70 (1995) $310-338$.

[9] A. Freire, Uniqueness for the harmonic map flow in two dimensions. Calc. Var. Partial Differential Equations 3 (1995) 95-105.

[10] F. Hülsemann and Y. Tourigny, A new moving mesh algorithm for the finite element solution of variational problems. SIAM J. Numer. Anal. 35 (1998) 1416-1438. 
[11] M. Pierre, Weak BV convergence of moving finite elements for singular axisymmetric harmonic maps. SIAM J. Numer. Anal. To appear.

[12] E. Polak, Algorithms and consistent approximations, Optimization, Applied Mathematical Sciences 124 (1997), SpringerVerlag, New York.

[13] J. Qing, On singularities of the heat flow for harmonic maps from surfaces into spheres. Comm. Anal. Geom. 3 (1995) $297-315$.

[14] S. Rippa and B. Schiff, Minimum energy triangulations for elliptic problems. Comput. Methods Appl. Mech. Engrg. 84 (1990) $257-274$.

[15] M. Struwe, The evolution of harmonic maps, in Proceedings of the International Congress of Mathematicians, Vol. I, II (Kyoto, 1990). Math. Soc. Japan (1991) 1197-1203.

[16] P. Topping, Reverse bubbling and nonuniqueness in the harmonic map flow. Internat. Math. Res. Notices 10 (2002) 505-520.

To access this journal online: www.edpsciences.org 